\title{
The evolution and refinement of vasoepididymostomy techniques
}

\author{
Peter T Chan
}

Obstructive azoospermia secondary to epididymal obstruction can be corrected by microsurgical reconstruction with vasoepididymostomy (VE). Although alternative management such as epididymal or testicular sperm aspiration in conjunction with intracytoplasmic sperm injection is feasible, various studies have established the superior cost-effectiveness of VE as a treatment of choice. Microsurgical VE is considered one of the most technically challenging microsurgeries. Its success rate is highly dependent on the skills and experience of the surgeons. Various techniques have been described in the literature for VE. We have pioneered a technique known as longitudinal intussusception VE (LIVE) in which the epididymal tubule is opened longitudinally to obtain a larger opening to allow its tubular content to pass through the anastomosis. Our preliminary data demonstrated a patency rate of over $90 \%$. This technique has been widely referenced in the recent literature including robotic-assisted microsurgery. The history of the development of different VE approaches, the preoperative evaluation along with the techniques of various VE will be described in this article.

Asian Journal of Andrology (2013) 15, 49-55; doi:10.1038/aja.2012.80; published online 19 November 2012

Keywords: longitudinal intussusception vasoepididymostomy (LIVE); intracytoplasmic sperm injection (ICSI); male infertility; microsurgery; obstructive azoospermia; vasoepididymostomy (VE)

\section{INTRODUCTION}

Though azoospermia represents a severe form of male factor infertility to manage, it can be caused by obstruction which can be corrected by surgical reconstruction. Indeed, azoospermia may be due to bilateral obstruction at any point of the male excurrent ductal system which comprises the efferent ductules, epididymis, vas deferens and the ejaculatory ducts. Primary obstructive azoospermia is most commonly due to bilateral obstruction of the epididymides, if the iatrogenic cause of vasal obstruction postvasectomy is excluded. Obstructive azoospermia due to epididymal obstruction can be corrected by microsurgical reconstruction with vasoepididymostomy (VE).

Most experts would agree that VE is the most technically challenging operation in male reproductive microsurgery. The history of the development of the surgical techniques for VE was reviewed by Chan et al. ${ }^{1,2}$ The earliest report of VE was attempted in 1903 by creation of a fistulous communication between the multiply incised epididymal tubules and the opened lumen of the vas deferens. Lespinasse in $1918^{3}$ was the first to attempt a precise epididymal tubule anastomosis to the vasal lumen. Prior to the introduction of microsurgical technique, however, the success rate of VE varied but was generally poor, both for patency and for subsequent pregnancy. With the introduction of optical enhancement, microsurgical end-to-end single tubule anastomosis was introduced by Silber in $1978,{ }^{4}$ and end-to-side anastomosis by Wagenknecht et al. ${ }^{5}$ and popularized by Thomas. ${ }^{6}$ The patency rate of VE using these conventional microsurgical techniques ranged from $50 \%$ to $85 \%$. Extreme precision and exquisite microsurgical skills are required to anastomose the delicate epididymal tubule, which is of a diameter of $150-250 \mu \mathrm{m}$, to the vasal lumen. The outcomes of these techniques are highly dependent on the experience of the surgeons. The use of an operating microscope is mandatory. Surgical loupes, which usually have a limit of magnification at $\times 6$, are not powerful enough to allow proper visualization of the epididymal tubule for precise placement of the microsutures.

Stefanovic et al. ${ }^{7}$ described a tubular intussusception for VE in rats using a single mucosal suture. Berger ${ }^{8}$ applied this technique in human, using three double-armed microsutures placed to an epididymal tubule in a triangular fashion (Figure 1). Upon opening the tubule, the three double-armed sutures are placed inside out through the vasal mucosa, yielding a six-point anchor for the anastomosis and allowing the epididymal tubule to intussuscept into the vasal lumen. Preliminary results of this three-suture triangulation intussusception technique were superior to previous techniques of microsurgical VE. Subsequently, Marmar ${ }^{9}$ modified this technique, using only two microsutures placed perpendicular to the epididymal tubule for the anastomosis (Figure 2).

Chan et al. ${ }^{1,2,10}$ reported recently that placing the two microsutures longitudinally to the epididymal tubules allows the incision on the tubules to be made longitudinally, resulting in a larger lumen of epididymal inflow to the anastomosis. Results in an animal study demonstrated superior patency rates of this microsurgical longitudinal intussusception VE (LIVE) compared to the original technique using three sutures or the modified technique using two sutures placed perpendicular to the epididymal tubules. This last modification with LIVE greatly simplifies the technique of microsurgical VE while allowing a superior patency rate to be achievable. We will describe the technique of LIVE in further details below. 


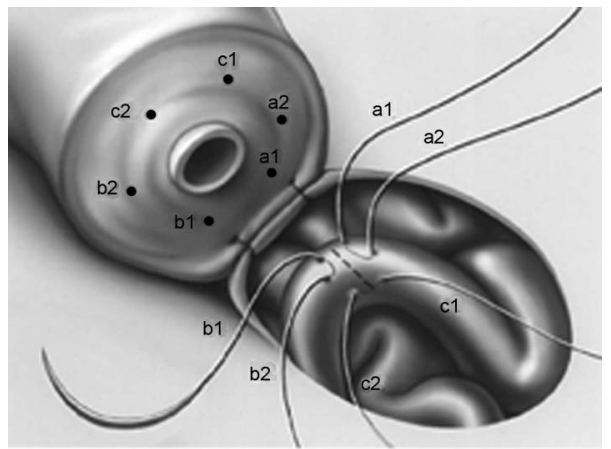

Figure 1 Triangulation intussusception end-to-side vasoepididymostomy (VE). Three double-armed microsutures are placed in a triangulation fashion on the epididymal tubule which is opened in the center of the triangle formed. The ends of the sutures are placed on the vasal end to complete the anastomosis.

\section{EVALUATIONS AND DIAGNOSIS}

A thorough history and physical examination often provide important clues that can lead to the diagnosis of epididymal obstruction. In North America, by far the most common cause of epididymal obstruction is prolonged vasal obstruction after vasectomy. Most investigators believe that after a vasectomy, the chronic pressure increase intraluminally within the excurrent ductal system leads to a 'blowout' injury in the epididymal tubules resulting in obstruction.

Several factors may help predict the risk of epididymal obstruction postvasectomy. On physical examination, the presence of a vasal sperm granuloma, which acts as a 'pop-off' valve to vent the intraluminal pressure, on the testicular end of the vas at the vasectomy site, decreases the risk of epididymal injury. The length of the vasal 'chimney' at the testicular end, as measured from the vasal-epididymal junction to the point of vasectomy, is another important finding on physical examination. Vasectomy performed in the proximal convoluted vas results in a shorter vasal 'chimney' than vasectomy performed in the straight vas. In the former case, the luminal pressure may be higher, resulting in a higher likelihood for subsequent epididymal injury leading to obstruction. The time since the vasectomy also correlates with the likelihood of epididymal obstruction. ${ }^{11-16}$ Most investigators believe that vasectomy performed more than 5 years, particularly those without a vasal sperm granuloma or a long vasal chimney, has a significant likelihood of requiring a VE on at least one side.

Parekattil et al. ${ }^{11,17}$ proposed a mathematical model to predict the need of VE for vasectomy reversal. The equation for the model is VE prediction score $=($ Age $\times 0.31)+($ Obstructive interval $\times 0.94)$. If the prediction score is greater than 20 , then a VE on at least one side is predicted. If the score is less than 20, a bilateral vasovasostomy is predicted. This predictor model has been validated in a multi-institutional study with an $84 \%$ sensitivity and $58 \%$ specificity for detecting patients who may require VE during a vasectomy reversal, ${ }^{11}$ though other authors have raised concern that this model might not be universally applicable. ${ }^{18}$ VE can be a much more technically challenging procedure than a vasovasostomy. Some investigators hold the opinion that microsurgeons attempting vasectomy reversal for cases with a significant likelihood of epididymal obstruction should be proficient in performing a $\mathrm{VE}^{19}$ to provide the best possible outcomes for patients.

Besides a vasectomy, other clinical clues in the patients' history that may suggest an obstruction of the excurrent ductal system of the male reproductive tract include previous surgeries, instrumentation or trauma in the groin, pelvis, scrotum, prostate and urethra. A family history of cystic fibrosis suggests that the patient may be a carrier of

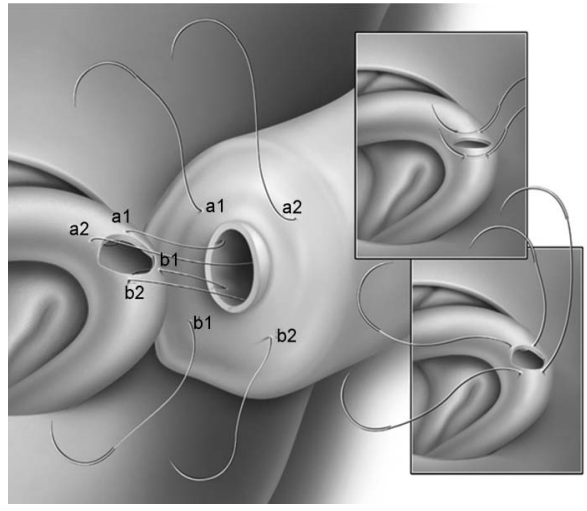

Figure 2 Two-needle intussusception vasoepididymostomy (VE). This technique allows the use of two double-armed sutures to provide a four-point fixation on the vasal end for the anastomosis.

mutations in the cystic fibrosis transmembrane conductance regulator gene and may present with congenital bilateral absence of vas deferens with epididymal obstruction. Infection such as remote epididymoorchitis may result in significant inflammation in the epididymis leading to obstruction. Patients may not always report or recall a remote episode of such an infection or they may have mistaken it as a simple urinary tract infection. Cases of the so-called idiopathic primary epididymal obstruction may have a history of lower urinary tract infection when a thorough history is taken.

Additional clues on physical examination that may suggest epididymal obstruction in an azoospermic patient include a full epididymis or epididymal cysts with normal testicular volume and texture. Congenital bilateral absence of vas deferens on scrotal examination represents another finding consistent with obstruction of the excurrent ductal system. These patients typically have low ejaculation volume and a semen $\mathrm{pH}$ below 7 , consistent with the seminal vesicle atresia that typically accompanies congenital bilateral absence of vas deferens. This is in contrast to patients with isolated bilateral epididymal obstruction, in whom semen analysis classically shows normal volume, fructose-positive azoospermia with $\mathrm{pH}>7$.

The diagnoses of unilateral obstruction and partial epididymal obstruction are difficult to make or confirm, as these patients generally do not present with azoospermia, but rather, with infertility with a combination of oligo-, astheno- and terato-spermia. If unilateral or partial epididymal obstruction is suspected clinically and VE or other surgical reconstruction of the excurrent ductal system are contemplated, cryopreservation of sperm preoperatively should be considered as it is possible that the semen profile may decline significantly postoperatively if the reconstructive surgery fails.

It should be noted that epididymal obstruction is generally an intraoperative diagnosis as suggested by the presence of active spermatogenesis within the testis and the absence of sperm in a patient's vas deferens. Although additional evaluations including cystoscopy and various imaging studies including scrotal and transrectal ultrasound, computer tomography and magnetic resonance imaging may provide information that is consistent with epididymal obstruction, they are neither sensitive or specific adequately enough to diagnose epididymal obstruction and are generally not required prior to VE.

Active spermatogenesis must be confirmed prior to attempting reconstruction of the excurrent ductal system. Men who have proven fecundity prior to the vasectomy, in the absence of additional male factor infertility, generally have a serum follicle-stimulating hormone in the low normal range and a normal testosterone level consistent 
with the presence of active spermatogenesis. In cases of idiopathic or primary epididymal obstruction in a man with no proven fecundity in history, confirmation of active spermatogenesis is necessary prior to proceeding to VE, particularly when the serum follicle-stimulating hormone level is in or above the high normal range. Confirmation of active spermatogenesis can be obtained through bilateral testicular biopsy performed as an isolated procedure ahead of time. Histologically, the cross-section of each seminiferous tubule should have over 20 mature spermatids. Alternatively, cytological examination of testicular aspiration for spermatozoa intraoperatively prior to VE may confirm the presence of active spermatogenesis.

\section{INDICATIONS FOR VE}

Reconstruction of the excurrent ductal system with VE is indicated in cases of obstructive azoospermia due to epididymal obstruction. This includes scenarios such as postepididymitis azoospermia, iatrogenic epididymal injury (e.g. posthydrocelectomy or orhiopexy), trauma or secondary epididymal obstruction due to prolonged obstruction of other parts of the excurrent ductal system (e.g. postvasectomy) leading to 'blow-out' epididymal tubular injury from chronic increase in the intraluminal pressure of the epididymal tubules.

VE should be performed in epididymal tubules that contain abundant sperm (motile or immotile) or sperm parts (heads and tails). Absence of abundant sperm or sperm parts indicates that the level of epididymal obstruction is located more proximally where the anastomosis should be performed. VE should not be attempted in the following situations. First, in the absence of vasa for anastomosing to the epididymides, or when the vasal gap is too big to be bridged despite appropriate surgical maneuvers for tissue mobilization, VE will not be feasible. Second, in case of azoospermia due to testicular failure (nonobstructive azoospermia), VE should not be attempted as no sperm can be found in the epididymis for the anastomosis to be successful. Although reconstruction is feasible for preepididymal obstruction at the level of the efferent ductules using similar techniques as VE, higher level of obstruction at the rete testis (as confirmed by the presence of active spermatogenesis in testicular biopsy but no sperm found at any level of the epididymal tubules or at the efferent ductules) cannot be successfully bypassed microsurgically.

\section{ALTERNATIVE MANAGEMENT OPTIONS}

Besides microsurgical reconstruction with VE, men with azoospermia due to epididymal obstruction may have sperm retrieved surgically from the epididymides (microsurgical epididymal sperm aspiration or percutaneous epididymal sperm aspiration) or from the testes (testicular sperm aspiration or testicular sperm extraction) for intracytoplasmic sperm injection (ICSI) to achieve pregnancy with their partners. With the increasing popularity and availability of assisted reproductive technologies such as in vitro fertilization and ICSI, the safety and efficacy of these technologies have improved significantly in the recent years. Particularly for couples in whom the female partners are in advanced reproductive age or have significant female factor infertility, sperm retrieval combined with ICSI can be an effective alternative to allow them to conceive as early as possible. However, it should be noted that upfront assisted reproduction combined with surgical sperm retrieval is an expensive option. Cost analyses performed by various investigators have consistently demonstrated that for men who have obstructive azoospermia, surgical reconstruction remains a more cost-effective option compared to upfront assisted reproduction, ${ }^{20-26}$ particularly if the couples have a low willingness to pay. ${ }^{24}$ In the hands of experienced microsurgeons, patency rate of over $90 \%$ can be achieved with microsurgical VE. We advocate the option of microsurgical sperm retrieval from the epididymal tubules during a VE for sperm cryopreservation. Should the VE fail, this option allows the couples to use the cryopreserved sperm for ICSI early on without requiring additional surgeries. Thus, proper counseling to the couples on the pros and cons of the various options available to them is essential to allow them to make the most appropriate therapeutic options to manage their infertility.

\section{SURGICAL TECHNIQUES}

A light general anesthesia is preferred. Slight movements are greatly magnified by the operating microscope and may disturb performance of the anastomosis. In the absence of any clinical evidence of complicated obstruction (e.g. multiple previous failure of reconstruction attempts, large vasal gap and significant fibrosis in scrotal structure on physical examination), regional anesthesia with sedation can be employed in cooperative and motivated patients. Appropriate intraoperative intravenous antibiotics should be considered particularly in cases with a significant past history of infection in the genito-urinary tract.

\section{Preparation of the vas}

A high scrotal incision (Figure 3 ) is preferred to allow adequate mobilization of the inguinal portion of the vas to anastomose to the epididymis without any tension. When isolating the vas, its periadventitial sheath should not be stripped off to preserve its blood supply. As the epididymis lies laterally in the posterior aspect of the testis, the vas should be mobilized and isolated lateral to the rest of the spermatic cord to allow a more direct contact with the epididymis for the anastomosis. In patients who had a previous vasectomy, the vas should be transected at the vasectomy site to evaluate whether the testicular vas contains sperm and whether a VE is indicated. The cut surface of the testicular end of the vas deferens is inspected with the operating microscope under $\times 15-\times 25$ magnification. A healthy, white mucosal ring should be seen that springs back immediately after gentle dilation with a $2-3 \mathrm{~mm}$ microvessel dilator. The muscularis should appear smooth and soft. A gritty-looking muscularis layer indicates the presence of scar/fibrotic tissues. Healthy bleeding should be noted from both the cut edge of the mucosa as well as the surface of the muscularis. If the blood supply is poor or the muscularis is gritty, the vas should be recut until healthy tissue is found.

In patients with primary epididymal obstruction with no previous vasectomy, the vas is isolated at the junction of the straight and convoluted vas where it is hemitransected with a $15^{\circ}$ ophthalmic knife. Transection of the vas at this area will allow the maximal length of straight vas to be preserved to allow a tension free VE to be performed.

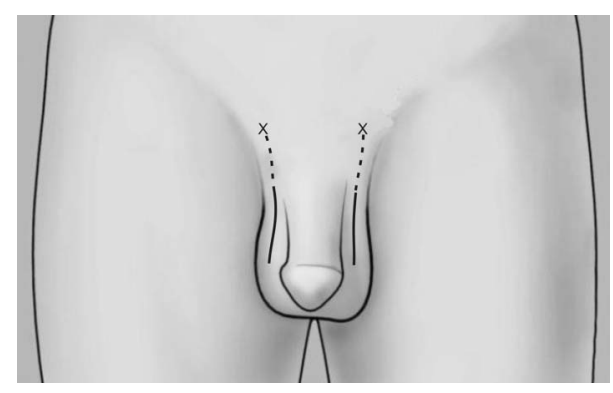

Figure 3 A high scrotal incision (solid lines) for vasoepididymostomy (VE) allows option to extend the incision (dotted lines) towards the external inguinal ring (marked by ' $X$ ') for mobilization of the abdominal vas to bridge a larger gap for the anastomosis. 
The vasal fluid is sampled and evaluated under light microscopy. The absence of sperm and sperm parts in the vasal fluid indicates epididymal obstruction. On the other hand, the presence of abundant motile sperm indicates the absence of epididymal obstruction and evaluation of vasal patency distally is essential to identify the location of the obstruction. In this case, the hemitransected vas can be reapproximated with interrupted 10-0 and 9-0 microsutures in two layers.

Patency of the abdominal end of the vas should be confirmed by saline vasogram in which a $24-\mathrm{G}$ soft angiocathter connected to a syringe containing $1-3 \mathrm{ml}$ of saline is used to cannulate the vas. Easy injection of saline towards the abdominal vas confirms patency. If patency is not certain, a dye-vasogram using 1-3 $\mathrm{ml}$ of $1: 10$ diluted indigo carmine should be performed. After injection of dye, bladder catheterization should review blue or green dye in the urine to confirm patency. If patency is not confirmed, formal vasography with diluted water-soluble radiographic contrast medium can be used to locate the obstruction.

\section{Identifcation of the appropriate epididymal tubule}

After confirming that a VE is necessary and that the abdominal vas is patent and of adequate length, the testis within the tunica vaginalis is delivered outside the wound. A longitudinal incision is made on the anterior aspect of the tunica vaginalis. In long-term epididymal obstruction, a small hydrocele is often noted when opening the tunica vaginalis. The epididymis is then inspected under the operating microscope at $\times 16-\times 25$ magnification to select an anastomotic site above the area of suspected obstruction. Often, a discrete yellow sperm granuloma is noted, above which there is indurated epididymis and dilated tubules, and below which the epididymis is soft and the tubules collapsed.

Selection of the correct level of the epididymal tubule for anastomosis should not be a random event. The diameter of epididymal tubule increases gradually from caput to cauda. Anastomosing to the larger tubule may result in a higher patency rate. ${ }^{27-30}$ Once a potential epididymal tubule for the anastomosis is identified, a relatively avascular area of the epididymal tunica is grasped with sharp jeweler forceps and tented upward. A 3-4 mm buttonhole is made in the tunica with microscissors to create a round opening that matches the outer diameter of the prepared vas deferens.

If the level of obstruction in the epididymis is not clearly demarcated, choose a tubule distally and puncture it with a 10-0 needle to aspirate epididymal fluid and examine it microscopically. If sperm are not found, proceed in an identical fashion proximally. Once sperm are identified, seal the puncture with a bipolar cautery and begin the anastomosis a few millimeters proximally along the epididymal tubule. Since the microsutures are to be placed longitudinally, a straight segment of the tubule is more preferable than one that is curved. This would allow the 'needle-bites' to run further along the tubule and a longer incision to be made longitudinally between the two needles.

\section{Preparation of the epididymal tubule}

The epididymal tunic covering the tubule is thick and tough. On the other hand, the bare epididymal tubule underneath is thin and delicate, and extra care should be taken to avoid accidentally puncture resulting in leakage of epididymal fluid and collapse of the tubule. After the initial cut on the epididymal tunic, there is often still a thin layer of residual tunic covering the epididymal tubule. Use of indigo carmine on the tissue may enhance visualization of the residual overlying tunic. The residual tunica should be dissected off fully with a combination of sharp and blunt dissection to expose the bare epididymal tubule underneath and to facilitate its intussusception into the vasal lumen when completing the anastomosis.
The vas deferens is drawn up through an opening in the tunica vaginalis and secured in proximity to the anastomotic site with two to four interrupted sutures of 6-0 polypropylene placed through the vasal adventitia and the tunica vaginalis. Care should be taken to avoid taking too deep a bite with the suture that may result in accidental closure of the vasal lumen, rendering failure of the surgery. The vasal lumen should reach the opening in the epididymal tunica easily with length to spare. To avoid tension or kinking of the vas, the surgeon should inspect the position and orientation of the vas not only when the testicle is delivered outside, but also when the testicle is placed back into the scrotum. The posterior edge of the epididymal tunica is then approximated to the posterior edge of the vas muscularis and adventitia with two to three interrupted sutures of 9-0 nylon (Figure 4a-4f). This is done in such a way as to bring the vasal lumen in close approximation to the epididymal tubule selected for anastomosis. Adequate hemostasis of the vas and the epididymal tunic is essential, as the fluid in the epididymis cannot dissolve blood clot which may obstruct the anastomosis.

\section{LIVE}

The LIVE is our preferred method of microsurgical VE as it greatly simplifies the procedure while yielding the highest patency rate among the various alternative techniques that we have previously employed. ${ }^{31-33}$ To allow even distribution of the suture points, four microdots are placed with a micromarking pen on the vasal ends to demarcate the exit points of the suture needles to be placed on the vas (Figure 4a). For the anastomosis, we prefer to use 1-inch monofilament $10-0$ nylon sutures, double-armed with $70-\mu \mathrm{m}$ diameter taper-point needles. Double-armed sutures allow inside-out placement of the needles on the mucosa, eliminating the need for manipulation of the mucosa and the possibility of back walling. Modification of the LIVE technique for the use of longer double-armed needles or single-armed needles will be described later.

The vas is secured with 9-0 nylon sutures on the edge of the tunica epididymis opening with vasal lumen positioned at the center of the selected epididymal tubule. Under the highest magnification of the operating microscope $(\times 25-\times 40)$, the needles of two double-armed 10-0 microsutures are placed longitudinally on the epididymal tubule (Figure 4b). The microneedles are left in the epididymal tubule until it is ready to be incised longitudinally. Since the diameter of the needle $(70 \mu \mathrm{m})$ is significantly larger than the suture diameter $(17 \mu \mathrm{m})$, pulling through the needle will result in leakage of the epididymal fluid.

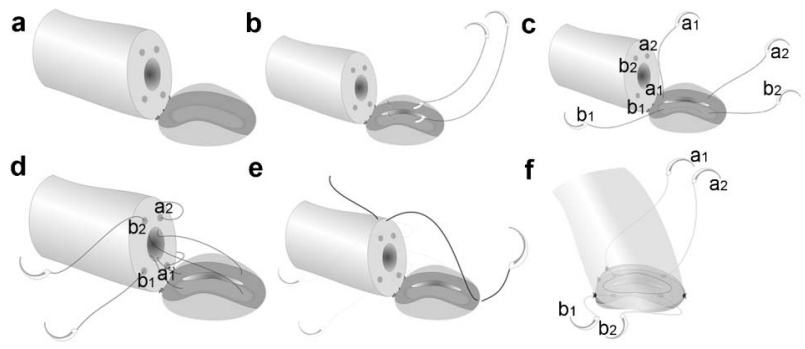

Figure 4 Longitudinal intussusception vasoepididymostomy (LIVE): (a) the vas is secured on the edge of the tunica epididymis with two to three 9-0 sutures with its lumen opposing the mid-portion of the isolated epididymal tubule; (b) placement of the two double-armed 10-0 mucosal sutures in a longitudinal fashion on the tubule which is opening with a longitudinal incision between the needles; $(\mathbf{c}, \mathbf{d})$ the sutures are pulled through and placed in an inside-out fashion on the vasal ends; (e,f) a 9-0 tension reducing suture is placed to position the vasal lumen in direct opposition with the epididymal lumen prior to tying the mucosal sutures. Both mucosal sutures can be tied to their own ends complete the anastomosis. 
The collapse of the epididymal tubule, along with the impaired visibility from the cloudy epididymal fluid, makes it difficult to place an additional suture or to make a precise opening in the tubule for the anastomosis.

Using a $15^{\circ}$ ophthalmic knife, the tubule is incised longitudinally between the two needles of the 10-0 sutures (Figure $4 \mathrm{~b}$ ). The epididymal fluid is then aspirated with $5 \mu$ micropipette or with a $24-\mathrm{G}$ angiocatheter connected to a syringe for aspiration. The fluid is examined for sperm under light microscopy. We generally recommend the surgeon to evaluate the epididymal fluid him- or herself with a bench top light microscope intraoperatively. This allows a quicker and more accurate answer to be obtained and avoids drying of the slide during transit to a pathologist/cytologist outside the operating room that may compromise the interpretation.

If motile sperm are found in the epididymal fluid, they may be cryopreserved for future use with ICSI in case the vasoepididymostomy is unsuccessful. Generally, if the level of the epididymal tubule is carefully selected, the fluid inside is likely to contain sperm or sperm parts. Absence of abundant sperm or sperm parts in the fluid indicates that obstruction is still proximal to the selected segment of tubule and anastomosis should not be performed there. The needles of the two double-armed 10-0 sutures are then pulled through and placed to the mucosa in the vasal ends in an inside-out fashion through the four microdots (Figure $4 \mathrm{c}$ and $4 \mathrm{~d}$ ).

\section{Completing the anastomosis}

To avoid tearing the 10-0 sutures out of the epididymal tubule during tying, it is necessary to position the vas to allow the vasal and epididymal lumina to be in direct opposition to each other. This is achieved by placing a 9-0 tension-reducing suture approximating the epididymal tunic to the vasal sheath prior to tying the 10-0 microsutures (Figure $4 \mathbf{e}$ and $4 \mathbf{f}$ ). This 9-0 suture is tied loosely to allow visualization of the anastomosis during tying of the 10-0 sutures.

The advantages of LIVE include providing a four-point fixation at the mucosal anastomosis using two double-armed microsutures and allowing a larger epididymal lumen to be created with a longitudinal incision on the tubule. A watertight outer layer anastomosis between the vasal sheath and the epididymal tunic is crucial to avoid leakage of fluid that can lead to formation of sperm granuloma. We recommend placing 10-12 interrupted sutures of 9-0 nylon for the outer layer closure. Care should be taken to avoid injury to the peripheral epididymal tubules during the placement of needles on the epididymal tunic.

LIVE is a delicate procedure. Errors can occur at several crucial points of the surgery, requiring the anastomosis to be performed more proximally. Examples may include absence of sperm or sperm parts in the epididymal fluid, tearing of the epididymal tubule or breaking a mucosal suture. In any of these cases, a new spot more proximally in the epididymis should be identified to redo the anastomosis. The surgeons should not feel frustrated when it is necessary to take down the set-up and redo the procedure at a more proximal site. Although it is time-consuming to redo an anastomosis, the extra time is well worth it. VE is a delicate operation where the outcome is highly dependent on technical perfection.

\section{Closure}

After careful hemostasis and copious irrigation of the tissue, the tunica vaginalis should be closed with absorbable sutures and the testis returned intrascrotally in the correct orientation. Care should be taken to avoid any tension when manipulating the testis and spermatic cord after a delicate anastomosis is completed. With careful control of hemostasis, the risk of scrotal edema or hematoma can be minimized and drainage is generally not necessary.

\section{Maneuvers to gain extra length to bridge a larger gap}

The simplest maneuver that should be attempted first to gain length to bridge a larger gap for the anastomosis is to mobilize the abdominal vas superiorly towards the external inguinal ring. The skin incision may be extended towards the external inguinal ring to facilitate this maneuver. Care should be taken to avoid stripping the periadventitial sheath to compromise the vasal blood supply. When there is still inadequate length of the vas deferens to reach the dilated epididymal tubule without tension, the cauda and corpus epididymis can be dissected off the testis and flipped up to obtain additional length (Figure 5). To do this, the full depth of the epididymis is encircled with a small Penrose drain at the level of obstruction and, under $\times 8$ 16 magnification, dissected distally off the testis, yielding sufficient length to perform the anastomosis. ${ }^{2,31}$ Usually, a surgical plane can be developed between the epididymis and testis, and injury to the epididymal blood supply can be minimized by staying right on the tunica albuginia of the testis. The inferior, and, if necessary, middle epididymal branches of the testicular artery are doubly ligated and divided to free an adequate length of epididymis. The superior-epididymal branches entering the epididymis at the caput are always preserved and can provide adequate blood supply to the entire epididymis. The tunica vaginalis is then closed over the testis with absorbable suture, which prevents drying of the testis and thrombosis of the surface testicular vessels during the anastomosis. The dissected epidididymis can remain outside the tunica vaginalis for the anastomosis.

\section{Robotic-assisted microsurgical VE}

With the increasing popularity and availability of surgical robots, there has been a simultaneous growth in the interest in adopting robotic surgical technologies in microsurgical VE. Early studies in animal mode ${ }^{34,35}$ using LIVE demonstrated a $100 \%$ patency rate. The group from Parekattil et al. ${ }^{36,37}$ has reported one of the earliest series of human robotic microsurgical VE. Using again LIVE, Parekettil et al. ${ }^{36,37}$ reported a significant decrease in the operating time with the robot (mean $120 \mathrm{~min}$; range 50-180 $\mathrm{min}$ ) compared to the non-robotic microsurgical method (150 $\mathrm{min}$; range $120-240 \mathrm{~min}$ ). They also reported an earlier return of sperm with the robotic technique compared to the non-robotic method, although the postoperative total motile sperm counts of the two methods were not significantly different.

Parekattil et al. ${ }^{37}$ noted several benefits of robotic surgical microsurgery: (i) a stable, ergonomic, scalable control system with threedimensional visualization and magnification; (ii) simultaneous ability to control three instruments and a camera; (iii) ability to integrate up to three visual inputs simultaneously in the surgeon console; and (iv) decreased fatigue and obviate the need for a skilled surgical assistant. Additional advantages of the robotic LIVE include improved stability and motion reduction during microsurgical suturing, ${ }^{34}$ which may be in part related to shorted operating time observed. ${ }^{37}$ It should, however, be pointed out that the availability of robotic is far from being universal in most parts of the world and that the cost-effectiveness of this approach remains to be established. Additional disadvantages such as

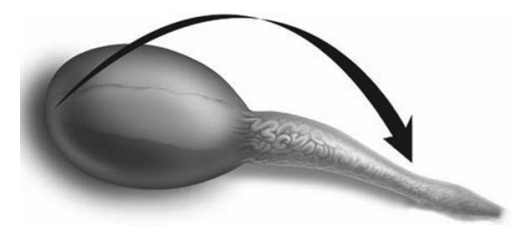

Figure 5 The corpus and caudal epididymis can be dissected off from the testis tunica albugenia and brought superiorly to bridge a massive vasal gap. 
visualization through video camera (albeit in three-dimensional and high definition) instead of direct optimal observation through surgical microscope (which most experts agree to be superior) and the lack of tactile response during tissue manipulation through the robotic arms may be overcome with further improvement in robotic surgical technologies.

\section{The use of single-armed microsutures for LIVE}

LIVE can be performed using 10-0 single-armed sutures which are less costly than the double-armed ones. ${ }^{38}$ The disadvantage is that the placement of two of the four needles on the vasal end has to be done in an outside-in fashion. Two 10-0 sutures are first placed on the vas in an outside-in fashion (Figure 6). Insertion of a 2-3 mm microvessel dilated during the outside-in placement of the needle may further decrease the risks of back walling of the vasal lumen mucosa. The LIVE procedure can then be performed in a similar fashion, with the two needles placed on the epididymal tubules where a longitudinal incision is made between the needles. The sutures are then pulled through and placed to the remaining two microdots marked on the vasal end in an inside-out fashion to complete the anastomosis. Tying of the two single-armed sutures can follow the same principle as described previously.

\section{The use of long double-armed microsutures for LIVE}

LIVE can be performed using a single long (6-inch) double-armed microsuture. Similar to the use of single-armed microsutures, the disadvantage is that the placement of two of the four needles on the vasal end has to be done in an outside-in fashion. The two needles at each end are first placed in the vas in an outside-in fashion. If the selected epididymal tubule lies perpendicularly to the vasal lumen, it is recommended to place the two needles on the two dots on the same side as the dominant hand of the surgeons (Figure 7). Insertion of a 2-3 $\mathrm{mm}$ microvessel dilated during the outside-in placement of the needle may further decrease the risks of back walling of the vasal lumen mucosa. The LIVE procedure can then be performed in a similar fashion, with the two needles first placed on the epididymal tubules where a longitudinal incision is made between the needles. The sutures are then pulled through and placed to the remaining two microdots marked on the vasal end in an inside-out fashion to complete the anastomosis. Since a single double-armed suture is used in this case, suture tying needs to be done only once. Care must be taken to ensure that there is no loose loop of suture before tying; otherwise, leakage of epididymal fluid may occur.

\section{OUTCOMES}

Studies on VE outcomes report patency rates between $31 \%$ and $92 \%$ and pregnancy rates between $10 \%$ and $50 \% .^{13,21,29,30,39-56}$ Most experts in this field agree that the technical experience of the surgeons is one of the most important factors for the success of VE. We reported our clinical experience of microsurgical LIVE in a series of 72 men with azoospermia due to epididymal obstruction. ${ }^{10}$ The mean age of the subjects was 39.3 years. The etiologies of obstruction were: postvasectomy $(69 \%)$, infection $(22 \%)$, iatrogenic $(5 \%)$, trauma $(1 \%)$ and idiopathic (3\%). The median duration of obstruction was 18.7 years. Previous failed attempts at reconstruction were noted in $38 \%$ of patients. Mean follow-up period was 16.3 months.

The patency rate, defined as more than 10000 sperm per $\mathrm{ml}$ of semen, at any time postoperatively, was $92 \%$. Early patency rate was achieved in $73 \%$ of subjects at $4-6$ weeks postoperatively. The median best sperm count was $12.9 \times 10^{6} \mathrm{ml}^{-1}$ with a $23 \%$ forward motility. The late 'shut-down' rate, defined as the percentage of subjects who had sperm postoperatively but later became persistently azoospermic was $4 \%$ at 1 year postoperatively. The median duration of the procedure was 55 min per anastomosis.

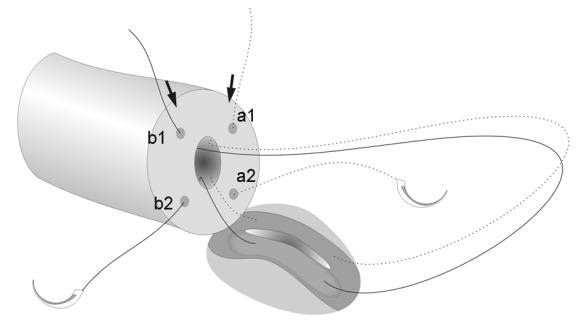

Figure 6 Placement of a single-armed microsuture for longitudinal intussusception vasoepididymostomy (LIVE). The arrows indicated the placement of the first bites of the two sutures.

Among patients with follow-up over 1 year, the natural pregnancy rate was $31 \%$. Median time to achieve natural pregnancy was 15.3 months. An additional 39\% of patients achieved pregnancy with assisted reproduction, all using fresh ejaculated sperm.

From our experience, LIVE greatly simplifies the technique for microsurgical VE while allowing a high patency rate of over $90 \%$ and an acceptable natural pregnancy rate to be achievable. Even when assisted reproductive technology is needed, fresh ejaculated sperm can be used without requiring a subsequent sperm retrieval procedure. Our clinical experience supports LIVE as an effective reconstruction approach for epididymal obstruction.

\section{COMPLICATIONS}

Potential surgical complications associated with VE include wound infection, scrotal edema, hematoma, orchalgia and persistent epididymal obstruction (surgical failure). Most of these complications are self-limiting and can be managed conservatively. More devastating complications such as ischemic epididymal fibrosis and testicular atrophy may be encountered rarely. The risks of these complications should be fully disclosed to all patients preoperatively. Patients should also be instructed on the various preventive measures. These include: i) avoidance of strenuous physical activities immediately after the surgery; ii) use of icepack for wound or scrotal compression to prevent the development of hematoma or edema; and iii) avoidance of ejaculation for up to 4 weeks to minimize the risks of disruption of the anastomosis due to the force of propulsion of the excurrent ductal system from orgasm.

The risk of developing these postoperative complications depends on the complexity of the surgery. In cases involving multiple previous unsuccessful reconstruction attempts with significant extent of tissue fibrosis in the scrotal contents, or in cases where additional dissection is required to mobilize the vas or the epididymis to bridge a larger gap for the anastomosis, the likelihood of developing complications would increase. With meticulous surgical techniques and adherence to the surgical principles discussed above, however, the risks of developing most of these complications can be minimized or avoided. The placement of an intrascrotal penrose drain secured to the

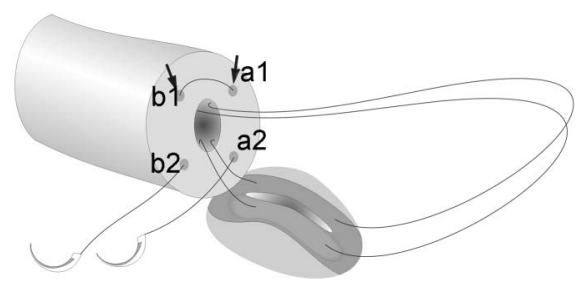

Figure 7 Placement of a long double-armed microsuture for longitudinal intussusception vasoepididymostomy (LIVE). The arrows indicated the placement of the first bites of the two needles. 
scrotal skin covered with an absorptive dressing may sometimes be used to minimize the risks of developing scrotal hematoma. Such a drain could be removed after $48 \mathrm{~h}$ if there is minimal amount of edema developed and the volume of drainage is minimal.

Postoperatively, semen analyses may be performed at 1 month and subsequently every 2-3 months. Patients who have motile sperm return to the ejaculate should consider cryopreserving sperm, as initially patent anastomoses may eventually shut down. From our experience with LIVE, the great majority of patients who have a patent anastomosis will have sperm in the ejaculate in the first 6 months. It is rare for patients who have been persistent azoospermic in the first 6 months postoperatively to have the anastomoses opened up. Persistently azoospermic men without cryopreserved sperm can opt for a redo VE or surgical sperm retrieval by various techniques combined with ICSI to achieve pregnancy with their partner.

\section{COMPETING FINANCIAL INTERESTS}

The author of this article declared no competing financial interests.

1 Chan PT, Li PS, Goldstein M. Microsurgical vasoepididymostomy: a prospective randomized study of 3 intussusception techniques in rats. J Urol 2003; 169: 1924-9.

2 Chan PT. Vasoepididymostomy. In: Graham S, Keane T, editors. Glenn's Urologic Surgery. 7th ed. Philadelphia, PA: Lippincott Williams \& Wilkins. 2009; p379-86.

3 Lespinasse VD. Obstructive sterility in the male treatment by direct vasoepididymostomy. JAMA 1918; 70: 448-50.

4 Silber SJ. Microscopic vasoepididymostomy: specific microanastomosis to the epididymal tubule. Fertil Steril 1978; 30: 565-71.

5 Wagenknecht LV, Klosterhalfen H, Schirren C. Microsurgery in andrologic urology. I. Refertilization. J Microsurg 1980; 1: 370-6.

6 Thomas AJ Jr. Vasoepididymostomy. Urol Clin North Am 1987; 14: 527-38.

7 Stefanovic KB, Clark SA, Buncke HJ. Microsurgical epididymovasostomy by tubule intussusception: a new technique in rat model. Fertil Steril 1991; 55: 189-93.

8 Berger RE. Triangulation end-to-side vasoepididymostomy. J Urol 1998; 159: 1951-3.

9 Marmar JL. Modified vasoepididymostomy with simultaneous double needle placement, tubulotomy and tubular invagination. J Urol 2000; 163: 483-6.

10 Chan PT, Lee R, Li PS, Libman J, Goldstein M. Six years of experience with microsurgical longitudinal intussusception vasoepididymostomy (LIVE): a prospective analysis. Proceedings of the 2008 Annual Meeting of the American Urological Association; 17-22 May 2008; Orlando, FL, USA. Abstract.

11 Parekattil SJ, Kuang W, Kolettis PN, Pasqualotto FF, Teloken P et al. Multiinstitutional validation of vasectomy reversal predictor. J Urol 2006; 175: 247-9.

12 Belker AM, Thomas AJ Jr, Fuchs EF, Konnak JW, Sharlip ID. Results of 1,469 microsurgical vasectomy reversals by the Vasovasostomy Study Group. J Urol 1991; 145: 505-11.

13 Kolettis PN, Sabanegh ES, D'Amico AM, Box L, Sebesta M et al. Outcomes for vasectomy reversal performed after obstructive intervals of at least 10 years. Urology 2002; 60: 885-8.

14 Kolettis PN, D'Amico AM, Box L, Burns JR. Outcomes for vasovasostomy with bilateral intravasal azoospermia. J Androl 2003; 24: 22-4.

15 Fuchs EF, Burt RA. Vasectomy reversal performed 15 years or more after vasectomy: correlation of pregnancy outcome with partner age and with pregnancy results of in vitro fertilization with intracytoplasmic sperm injection. Fertil Steril 2002; 77: 516-9.

16 Silber SJ. Reversal of vasectomy and the treatment of male infertility. Role of microsurgery, vasoepididymostomy, and pressure-induced changes of vasectomy. Urol Clin North Am 1981; 8: 53-62.

17 Parekattil SJ, Kuang W, Agarwal A, Thomas AJ. Model to predict if a vasoepididymostomy will be required for vasectomy reversal. J Urol 2005; 173: 1681-4.

18 Kavoussi PK, Bird ET. Validation of a vasoepididymostomy predictor model: is vasoepididymostomy truly predictable preoperatively? Fertil Steril 2009; 92: 180-1.

19 Chawla A, O'Brien J, Lisi M, Zini A, Jarvi K. Should all urologists performing vasectomy reversals be able to perform vasoepididymostomies if required? J Urol 2004; 172: 1048-50

20 Pavlovich CP, Schlegel PN. Fertility options after vasectomy: a cost-effectiveness analysis. Fertil Steril 1997; 67: 133-41.

21 Kolettis PN, Thomas AJ. Vasoepididymostomy for vasectomy reversal: a critical assessment in the era of intracytoplasmic sperm injection. J Urol 1997; 158: 467-70.

22 Donovan JF Jr, DiBaise M, Sparks AE, Kessler J, Sandlow JI. Comparison of microscopic epididymal sperm aspiration and intracytoplasmic sperm injection/invitro fertilization with repeat microscopic reconstruction following vasectomy: is second attempt vas reversal worth the effort? Hum Reprod 1997; 13: 387-93.

23 Meng MV, Greene KL, Turek PJ. Surgery or assisted reproduction? A decision analysis of treatment costs in male infertility. J Urol 2005; 174: 1926-31.
24 Hsieh MH, Meng MV, Turek PJ. Markov modeling of vasectomy reversal and ART for infertility: how do obstructive interval and female partner age influence cost effectiveness? Fertil Steril 2007; 88: 840-6.

25 Lee R, Li PS, Goldstein M, Tanrikut C, Schattman G et al. A decision analysis of treatments for obstructive azoospermia. Hum Reprod 2008; 23: 2043-9.

26 Lee R, Li PS, Schlegel PN, Goldstein M. Reassessing reconstruction in the management of obstructive azoospermia: reconstruction or sperm acquisition? Urol Clin North Am 2008; 35: 289-301.

27 Schoysman RJ, Bedford JM. The role of the human epididymis in sperm maturation and sperm storage as reflected in the consequences of epididymovasostomy. Fertil Steril 1986; 46: 293-9.

28 Takihara $\mathrm{H}$. The treatment of obstructive azoospermia in male infertility-past, present, and future. Urology 1998; 51(5A Suppl): 150-5.

29 Zhang GX, Bai WJ, Xu KX, Wang XF, Zhu JC. Clinical observation of loupe-assisted intussusception vasoepididymostomy in the treatment of obstructive azoospermia (analysis of 49 case reports). Asian J Androl 2009; 11: 193-9.

30 Peng J, Yuan Y, Zhang Z, Gao B, Song W et al. Patency rates of microsurgical vasoepididymostomy for patients with idiopathic obstructive azoospermia: prospective analysis of factors associated with patency-single-center experience. Urology 2012; 79: 119-22.

31 Chan PT, Goldstein M. Vasectomy and vasectomy reversal. In: Kandeel FR, editor Male Reproductive Dysfunction. New York: Informa Healcare; 2007. p385-405.

32 Chan PT, Goldstein M. Reproductive tract reconstruction and vasectomy reversal. In Chan PT, Goldstein M, Rosenwaks Z, editors. Reproductive Medicine Secrets. Philadelphia, PA: Hanley \& Belfus; 2004. p112-35.

33 Chan PT, Brandell RA, Goldstein M. Prospective analysis of outcomes after microsurgical intussusception vasoepididymostomy. Br J Urol Int 2005; 96: 598-601.

34 Schiff J, Li PS, Goldstein M. Robotic microsurgical vasovasostomy and vasoepididymostomy: a prospective randomized study in a rat model. J Urol 2004; 171: 1720-5.

35 Schiff J, Li PS, Goldstein M. Robotic microsurgical vasovasostomy and vasoepididymostomy in rats. Int J Med Robot 2005; 1: 122-6.

36 Parekattil SJ, Gudeloglu A, Brahmbhatt J, Wharton J, Priola KB. Robotic assisted versus pure microsurgical vasectomy reversal: technique and prospective database control trial. J Reconstr Microsurg 2012; 28: 435-44.

37 Parekattil SJ, Brahmbhatt JV. Robotic approaches for male infertility and chronic orchialgia microsurgery. Curr Opin Urol 2011; 21: 493-9.

38 Monoski MA, Schiff J, Li PS, Chan PT, Goldstein M. Innovative single-armed suture technique for microsurgical vasoepididymostomy. Urology 2007; 69: 800-4.

39 McLoughlin MG. Vasoepididymostomy: the role of the microscope. Can J Surg 1982; 25: 41-3.

40 Dubin L, Amelar RD. Magnified surgery for epididymovasostomy. Urology 1984; 23: 525-8.

41 Fogdestam I, Fall M, Nilsson S. Microsurgical epididymovasostomy in the treatment of occlusive azoospermia. Fertil Steril 1986; 46: 925-9.

42 Lee HY. A 20-year experience with epididymovasostomy for pathologic epididyma obstruction. Fertil Steril 1987; 47: 487-91.

43 Fuchs EF. Restoring fertility through epididymovasostomy. Contemp Urol 1991; 3 : 27-31.

44 Schlegel PN, Goldstein M. Microsurgical vasoepididymostomy: refinements and results. J Urol 1993; 150: 1165-8.

45 Matsuda T, Horii Y, Muguruma K, Komatz Y, Yoshida O. Microsurgical epididymovasostomy for obstructive azoospermia: factors affecting postoperative fertility. Eur Urol 1994; 26: 322-6.

46 Matthews GJ, Schlegel PN, Goldstein M. Patency following microsurgical vasoepididymostomy and vasovasostomy: temporal considerations. J Urol 1995 154: 2070-3.

47 Jarow JP, Sigman M, Buch JP, Oates RD. Delayed appearance of sperm after end-toside vasoepididymostomy. J Urol 1995; 153: 1156-8.

48 Boeckx W, van Helden S. Microsurgical vasoepididymostomy in the treatment of occlusive azoospermia. Br J Urol 1996; 77: 577-9.

49 Berardinucci D, Zini A, Jarvi K. Outcome of microsurgical reconstruction in men with suspected epididymal obstruction. J Urol 1998; 159: 831-4.

50 Hibi H, Yamada $\mathrm{Y}$, Honda N, Fukatsu H, Katsuno $\mathrm{S}$ et al. Microsurgical vasoepididymostomy with sperm cryopreservation for future assisted reproduction. Int J Urol 2000; 7: 435-9.

51 Schrepferman CG, Carson MR, Sparks AE, Sandlow JI. Need for sperm retrieval and cryopreservation at vasectomy reversal. J Urol 2001; 166: 1787-9.

52 Schoor RA, Elhanbly SM, Ross LS, Niederberger CS. The influence of obstructive interval on patency rates following microsurgical epididymovasostomy. World J Urol 2002; 19: 453-6.

53 Smrkolj T, Virant-Klun I, Sinkovec J, Oblak C, Zorn B. Epididymovasostomy as the first-line treatment of obstructive azoospermia in young couples with normal spermatogenesis. Reprod Biomed Online 2010; 2: 594-601.

54 Ho KL, Wong MH, Tam PC. Microsurgical vasoepididymostomy for obstructive azoospermia. Hong Kong Med J 2009; 15: 452-7.

55 Kumar R, Mukherjee S, Gupta NP. Intussusception vasoepididymostomy with longitudinal suture placement for idiopathic obstructive azoospermia. J Urol 2010; 183: 1489-92.

56 Paick JS, Hong SK, Yun JM, Kim SW. Microsurgical single tubular epididymovasostomy: assessment in the era of intracytoplasmic sperm injection. Fertil Steril 2000; 74: 920-4. 